\title{
Topology Optimization of Structure for Dynamic Properties Considering Hybrid Uncertain Parameters
}

\author{
$\mathrm{ZC} \mathrm{He} e^{\mathrm{a}, \mathrm{c}}, \mathrm{Y} \mathrm{Wu}{ }^{\mathrm{a}}$, Eric $\mathrm{Li}^{\mathrm{b}^{*}}$ \\ a State Key Laboratory of Advanced Design and Manufacturing for Vehicle Body, Hunan University, \\ Changsha, 410082 P. R. China \\ ${ }^{\mathrm{b}}$ Department of Mechanical and Automation Engineering, The Chinese University of Hong Kong, Shatin, \\ NT, Hong Kong, China \\ ${ }^{\mathrm{c}}$ The State Key Laboratory of Fluid Power and Mechatronic Systems, Zhejiang University, Hangzhou, \\ 310027, China
}

\begin{abstract}
In the design and manufacturing of mechanical components, the dynamic properties of continuum structure are one of the most significant performances. At the same time, the uncertainty is widespread in these dynamic problems. This paper presents a robust topology optimization methodology of structure for dynamic properties with consideration of hybrid uncertain parameters. The imprecise probability uncertainties including materials, geometry and boundary condition are treated as an interval random model, in which the probability distribution parameters of random variables are modeled as the interval variables instead of given precise values. Two dynamic properties, including dynamic-compliance and eigenvalue, are chosen as the objective function. In addition, different excitation frequency or eigenvalue is discussed. In this work, the bi-directional evolutionary structural optimization (BESO) method is adopted to find the optimal robust layout of the structure. A series of numerical examples is presented to illustrate the optimization procedure, and the effectiveness of the proposed method is demonstrated clearly.
\end{abstract}

Keywords: Robust topology optimization; dynamic-compliance; eigenvalue; hybrid uncertain

*Corresponding author. Tel./fax: +86 73188822051.

E-mail address:ericsg2012@gmail.com (Eric Li) 


\section{Introduction}

Topology optimization for continuum structures is one of the most general forms of structural optimization [1-5]. Since the late 1980s, enormous progress has been made in the theory, methods and applications of topology optimization [6]. On the basis of the topology optimization methods, the topology optimization for dynamic property, the one of the most important performance in designing of structure, was first proposed with the landmark work of Bendsøe and Kikuchi [7], and followed by a rapid expansion [8-14]. Diaz and Kikuchi [15] first studied the shape and topology optimization of structures to maximize a natural frequency using homogenization method. Ma et al. [13, 16] and Min et al. [17] applied the homogenization method to analyze the vibrating structures. Jog [18] studied the topology configuration of structures subjected to periodic loadings from the global and local dynamic constraints. Du and Olhoff [19] employed SIMP (Solid Isotropic Material with Penalization) to maximize the eigenvalue of higher order, or the gap between two consecutive Eigen-frequencies of given orders. Additionally, ESO (Evolutionary Structural Optimization) was employed to optimization problems with frequency constraints (Xie and Steven [20]) and dynamic loads (Huang et al [21]). Yan et al. [22] present a topology optimization method to optimize the plate structural dynamic performance.

However, these above algorithms are usually with the assumption that the system parameters are deterministic, which means that the dynamic performance of the continuum structure considering uncertainties of system parameters is ignored. However, in practical engineering, the parameters of system such as material 
properties, geometric properties, and boundary conditions might be uncertain, which leads to the optimization procedure cannot be perfect with just considering the deterministic parameters. It is worth noting that these errors and uncertainties are small in most cases, but coupling together can cause large deviations in the system, especially in dynamic problem, which is of high sensitivity.

Generally, probabilistic method [23-26], fuzzy-set method [27-29], interval method [30-35], and convex model [36-38] are the main ways to deal with uncertain parameters in the system. However, due to the complexity of the parameters and the disparate availability of uncertainty, the use of a single uncertainty modeling technology has been unable to meet the needs of simulation in the modern engineering system model [39-41]. For this reason, the hybrid approach, a method inspired by these uncertainty analyses, is proposed [40-47]. Among these hybrid uncertainty methods, the interval random method is a better choice when the probability distribution of the uncertainty can only be acquired from limited interval information [48]. The interval random model was firstly proposed by Elishakoff et al. $[49,50]$, and subsequently applied to the structural response analysis [51] and the structural reliability analysis [52-54].

In order to solve the problem of uncertainty, two structural optimization methods are adopted. One approach considers the possibility of structural failure and evaluates its performance through a so-called reliability index, which is known as reliability-based design optimization $[55,56]$; the other one is to find an optimal design that minimizes the effect of uncertain variables on the performance so that the 
design can be applied to all conditions. This method is called the robust design optimization $[57,58]$, which is considered in this paper. In the topology optimization, the robust design optimization can be extended to the robust topology optimization (RTO), and several algorithms have been proposed to handle uncertainties in topology optimization. Ben-Tal and Nemirovski [59] proposed a method based on semi-definite programming for robust truss topology optimization accounting for uncertain load conditions. Lógó et al. [60, 61] presented RTO algorithms using a first-order approximation for compliance in the presence of uncertainty in applied loads. Chen et al. $[62,63]$ studied a level-set based robust shape and topology optimization under random loading, material properties and geometrical uncertainties. Asadpoure et al. [64] proposed a method for robust structural topology optimization in the second order statistics uncertainties. Dunning et al. [65] formulated a robust topology optimization method that considered simultaneous minimization of expectancy and variance of compliance under uncertainty in loading magnitude. Schevenels et al. [66] focused on a robust topology optimization approach accounting for spatially varying manufacturing errors for the design of macro-, micro- or nano- structures. Zhao et al. [67] presented a convex modeling based topology optimization with load uncertainty. Chen et al. [68] carried out a robust topology optimization of structures with interval random parameters for static performance.

From the overall perspective, research on the dynamic property based on topology optimization considering the hybrid interval random variables, still has not been studied systematically and some important issues are still unsolved. Firstly, the 
dynamic property with uncertain parameters is an intricate target [69], which means that the performance of the system under various dynamic frequencies needs to be taken into account. Secondly, the eigenvalues of structure with uncertainty parameters, which is of great importance in many engineering fields, e.g. aerospace and automotive industries [20], should be considered. Finally, the optimization of dynamic performance is characterized by a high sensitivity that leads to some difficulties in the numerical processing. It is necessary to propose a numerical solution for dynamic performance topology optimization to overcome the high sensitivity of the dynamic problem. In short, it is necessary to develop an efficient, robust and accurate algorithm for the structural dynamic topology optimization with hybrid uncertainties.

In this paper, the hybrid interval random model is proposed to deal with the uncertainties in topology optimization for dynamic performance by using bi-directional evolutionary structural optimization (BESO) method. The dynamic-compliance and eigenvalues are the two optimization objectives of dynamic performance. This paper is outlined as follows: Section 2 illustrates the interval random variables and presents the numerical perturbation analysis model considering the interval random variables. Section 3 develops topology optimization formulations and sensitivity analysis for dynamic property. Section 4 elaborates the algorithm of RTO for dynamic property with interval random uncertainties. Section 5 describes the numerical implementation of the proposed BESO procedure for the topology optimization of structure of dynamic property with hybrid uncertainty parameters. Three numerical examples are presented in Section 6; and the final conclusions are 
made in Section 7.

\section{Interval random parameter model}

\subsection{Definition of interval random variable}

Two main steps are required to model the interval random parameters. First, a random distribution is used to describe the uncertainty for each uncertain parameter. Second, the probability distribution parameters are given by the intervals rather than the deterministic values, which are difficult to acquire from the limited information $[49,50]$. The interval random variables are represented to describe the existing uncertainties in material properties, geometric properties and boundary conditions. Let $\mathbf{r}(\mathbf{i})$ be the parameter vector of interval random variables, and $\mathbf{i}$ be the interval vector of every random parameter $[48,70,71]$. To implement the first step, $\mathbf{r}(\mathbf{i})$ can be describe as Eq. (1):

$$
\mathbf{r}(\mathbf{i})=\left(r_{1}(\mathbf{i}), r_{2}(\mathbf{i}), \ldots \ldots, r_{z}(\mathbf{i}), \ldots \ldots\right), \quad z=1,2, \ldots \ldots, J_{1}
$$

where $r_{z}(\mathbf{i})$ represents the $z$ th interval random parameter with an interval parameter $i_{y}$, and $z$ denotes the identifier of the random parameters. $J_{l}$ represents the number of random parameters. In this paper, the random parameter $r_{z}(\mathbf{i})$ is assumed to obey the normal distribution. The interval vector $\mathbf{i}$ can be expressed as follows:

$$
\mathbf{i}=\left[i_{1}, i_{2}, \ldots \ldots, i_{y}, \ldots \ldots .\right], \quad y=1,2, \ldots \ldots, J_{2}
$$

in which $i_{y}$ denotes the $y$ th interval parameter. The symbol $y$ denotes the identifier of the interval parameters. $J_{2}$ represents the number of the interval parameters. For every number of interval parameters ( 1 to $\left.J_{2}\right), i_{y}$ can be expressed as 


$$
\begin{aligned}
& i_{y}=\left[\underline{i_{y}}, \overline{i_{y}}\right]=i_{y}^{m}+\Delta i_{y}^{I}, \\
& i_{y}^{m}=\frac{i_{y}+\overline{i_{y}}}{2}, \quad \Delta i_{y}^{I}=\left[-\Delta i_{y},+\Delta i_{y}\right], \quad \Delta i_{y}=\frac{\overline{i_{y}}-\underline{\underline{y}}}{2}
\end{aligned}
$$

where $i_{y}$ and $\overline{i_{y}}$ denote the lower and upper bounds of interval parameter $i_{y} ; i_{y}^{m}$ represents the mean value of $i_{y}$, which can be calculated by averaging the lower and upper bounds value; $\Delta i_{y}^{I}$ denotes the uncertainty of the interval vector $i_{y}$, it can be obtained by averaging the difference of the lower and upper bounds value.

The expectation $\mu(\mathbf{r}(\mathbf{i}))$ and variance $\sigma^{2}(\mathbf{r}(\mathbf{i}))$ of the interval random vector can be expressed as

$$
\begin{aligned}
& \mu(\mathbf{r}(\mathbf{i}))=\mu\left(r_{1}(\mathbf{i}), r_{2}(\mathbf{i}), \ldots \ldots, r_{J_{l}}(\mathbf{i})\right) \\
& \sigma^{2}(\mathbf{r}(\mathbf{i}))=\sigma^{2}\left(r_{l}(\mathbf{i}), r_{2}(\mathbf{i}), \ldots \ldots, r_{J_{l}}(\mathbf{i})\right)
\end{aligned}
$$

\subsection{Perturbation analysis with interval random parameters}

To analyze the perturbation caused by the interval random parameters, the interval variables $\mathbf{i}$ of the interval random parameters $\mathbf{r}(\mathbf{i})$ can be first regarded as deterministic. In this case, the first-order Taylor expansion of the interval random parameter $\mathbf{R}(\mathbf{r}(\mathbf{i}))$ at the expectation of the interval random parameter $\mathbf{r}(\mathbf{i})$ can be expressed as

$$
\mathbf{R}(\mathbf{r}(\mathbf{i}))=\mathbf{R}(\mu(\mathbf{r}(\mathbf{i})))+\sum_{z} \frac{\partial \mathbf{R}(\mu(\mathbf{r}(\mathbf{i})))}{\partial r_{z}}\left(r_{z}-\mu\left(r_{z}(\mathbf{i})\right)\right)+R_{1}(\mathbf{r}(\mathbf{i}))
$$

As the variation of parameter is relatively small to itself, the error of the first-order Taylor expansion $R_{1}(\mathbf{r})$ in Eq. (5) can be ignored. It is obvious that, in Eq. (5), the interval random parameter $\mathbf{R}(\mathbf{r}(\mathbf{i}))$ is divided into two parts: the first part of Eq. 
(5) represents the expectation $\mathrm{E}(\mathbf{R})$, and the second part denotes the standard variance $\mathrm{SD}(\mathbf{R}) . \mathrm{E}(\mathbf{R})$ and $\mathrm{SD}(\mathbf{R})$ are stated as

$$
\begin{aligned}
& \mathrm{E}(\mathbf{R})=\mathbf{R}(\mu(\mathbf{r}(\mathbf{i}))) \\
& \mathrm{SD}(\mathbf{R})=\sqrt{\sum_{z}\left(\frac{\partial \mathbf{R}(\mu(r(\mathbf{i})))}{\partial r_{z}} \sigma\left(r_{z}(\mathbf{i})\right)\right)^{2}}
\end{aligned}
$$

Note that the interval variables in Eqs. (6) and (7) are regarded as deterministic. Considering the interval variables, the first-order Taylor expansion is adopted again at the mean value of the interval vector i. Eqs. (6) and (7) can be transformed to Eqs. (8) and (9), respectively:

$$
\begin{aligned}
& \mathrm{E}(\mathbf{R})=\mathbf{R}\left(\mu\left(\mathbf{r}\left(\mathbf{i}^{m}\right)\right)\right)+\sum_{z} \sum_{y} \frac{\partial \mathbf{R}\left(\mu\left(\mathbf{r}\left(\mathbf{i}^{m}\right)\right)\right)}{\partial \mu\left(r_{z}(\mathbf{i})\right)} \frac{\partial \mu\left(r_{z}\left(\mathbf{i}^{m}\right)\right)}{\partial \mathbf{i}_{y}}\left(\mathbf{i}_{y}-\mathbf{i}_{y}^{m}\right)+R_{1}(\mathbf{i}) \\
& =\mathbf{R}_{0}+\sum_{z} \sum_{y} \mathbf{R}_{1, z} \frac{\partial \mu\left(r_{z}(\mathbf{i})\right)}{\partial \mathbf{i}_{y}^{I}}\left(\mathbf{i}_{y}-\mathbf{i}_{y}^{m}\right)+R_{1}(\mathbf{i}) \\
& \mathrm{SD}(\mathbf{R})=\sqrt{\sum_{z}\left(\frac{\partial \mathbf{R}\left(\mu\left(r\left(\mathbf{i}^{m}\right)\right)\right)}{\partial r_{z}} \sigma\left(r_{z}\left(\mathbf{i}^{m}\right)\right)+\sum_{y}\left(\begin{array}{l}
\frac{\partial^{2} \mathbf{R}\left(\mu\left(\mathbf{r}\left(\mathbf{i}^{\mathrm{m}}\right)\right)\right)}{\partial r_{z} \partial i_{y}} \sigma\left(r_{z}\left(\mathbf{i}^{m}\right)\right) \Delta i_{y} \\
+\frac{\partial \mathbf{R}\left(\mu\left(\mathbf{r}\left(\mathbf{i}^{m}\right)\right)\right)}{\partial r_{z}} \frac{\partial \sigma\left(r_{z}\left(\mathbf{i}^{m}\right)\right)}{\partial i_{y}} \Delta i_{y}
\end{array}\right)+R_{1}(\mathbf{i})\right)^{2}} \\
& =\sqrt{\sum_{z}\left(\mathbf{R}_{2, z} \sigma\left(r_{z}\left(\mathbf{i}^{m}\right)\right)+\sum_{y}\left(\mathbf{R}_{3, z y} \sigma\left(r_{z}\left(\mathbf{i}^{m}\right)\right) \Delta i_{y}+\mathbf{R}_{2, z} \frac{\partial \sigma\left(r_{z}\left(\mathbf{i}^{m}\right)\right)}{\partial i_{y}} \Delta i_{y}\right)+R_{1}(\mathbf{i})\right)^{2}}
\end{aligned}
$$

when the variation of parameter is relatively small to itself, the error of the first-order Taylor expansion $R_{1}(\mathbf{r})$ in Eqs. (8) and (9) can be ignored. $\mathbf{R}_{0}, \mathbf{R}_{1, z}, \mathbf{R}_{2, z}$ and $\mathbf{R}_{3, z y}$ can be expressed by the following equation:

$$
\mathbf{R}_{0}=\mathbf{R}\left(\mu\left(\mathbf{r}\left(\mathbf{i}^{\mathrm{m}}\right)\right)\right)
$$




$$
\begin{aligned}
& \mathbf{R}_{1, z}=\frac{\partial \mathbf{R}\left(\mu\left(\mathbf{r}\left(\mathbf{i}^{m}\right)\right)\right)}{\partial \mu\left(r_{z}(\mathbf{i})\right)} \\
& \mathbf{R}_{2, z}=\frac{\partial \mathbf{R}\left(\mu\left(\mathbf{r}\left(\mathbf{i}^{m}\right)\right)\right)}{\partial r_{z}} \\
& \mathbf{R}_{3, z y}=\frac{\partial^{2} \mathbf{R}\left(\mu\left(\mathbf{r}\left(\mathbf{i}^{\mathrm{m}}\right)\right)\right)}{\partial r_{z} \partial i_{y}}
\end{aligned}
$$

\section{Topology optimization for dynamic performance}

In the design of structural dynamic performance, reducing the dynamic responses and raising the eigenvalues of structure are often considered. In this section, the above two dynamic performance-based topological optimization equations and sensitivity analysis are introduced. First of all, an alternative material interpolation scheme is presented.

\subsection{An alternative material interpolation scheme}

To obtain the gradient information of the design variable, a material interpolation scheme proposed by SIMP method [72] is usually adopted. However, such a scheme would result in numerical difficulties [73], which means, in dynamic problems, the high ratio between mass matrix and stiffness matrix could cause artificial localized vibration modals in low density regions. One effective method [19] to deal with this problem is to keep the ratio between mass and stiffness constant when $x_{\mathrm{e}}=x_{\min }$ by

$$
\begin{aligned}
& \rho\left(x_{\text {min }}\right)=x_{\text {min }} \rho^{0} \\
& E\left(x_{\text {min }}\right)=x_{\text {min }} E^{0}
\end{aligned}
$$

where $\rho^{0}$ and $E^{0}$ denote the density and Young's modulus of the solid materials, respectively. Therefore, an alternative material interpolation scheme can be expressed 
as:

$$
\begin{aligned}
& \rho\left(x_{e}\right)=x_{e} \rho^{0} \\
& E\left(x_{e}\right)=\left[\frac{x_{\min }-x_{\min }^{p}}{1-x_{\min }^{p}}\left(1-x_{e}^{p}\right)+x_{e}^{p}\right] E^{0} \quad\left(0<x_{\min } \leq x_{e} \leq 1\right)
\end{aligned}
$$

$\frac{\partial \mathbf{K}}{\partial x_{e}}$ and $\frac{\partial \mathbf{M}}{\partial x_{e}}$ can be directly obtained from Eq. (15) as

$$
\begin{aligned}
& \frac{\partial \mathbf{M}}{\partial x_{e}}=\mathbf{M}_{e}^{0} \\
& \frac{\partial \mathbf{K}}{\partial x_{e}}=\frac{1-x_{\min }}{1-x_{\min }^{p}} p x_{e}^{p-1} \mathbf{K}_{e}^{0}
\end{aligned}
$$

where $\mathbf{M}_{e}^{0}$ and $\mathbf{K}_{e}^{0}$ are mass and stiffness matrices of element $e$ when it is solid [74].

\subsection{Dynamic-compliance topology optimization}

It is generally desirable to minimize the overall response level of the structure in the design of the vibration and noise reduction, and the specific target values can be chosen differently. One of them is to optimize the steady-state response amplitude [12, 75].Another common approach is to introduce a concept which is widely used in static condition, the compliance, to dynamic field [20, 76-78], which is described below.

\section{Problem statement}

The dynamic-compliance optimization problem under dynamic external excitation can be expressed [79] as follows:

$$
\begin{gathered}
\min _{\rho_{e}, e=1, \ldots, N_{E}} C_{d}=\mathbf{F}^{\mathrm{T}} \mathbf{U}=\mathbf{U}^{\mathrm{T}} \mathbf{K}_{d} \mathbf{U} \\
\text { Subject to: } \mathbf{K}_{d} \mathbf{U} \equiv\left(\mathbf{K}-\omega_{p}^{2} \mathbf{M}\right) \mathbf{U}=\mathbf{F}
\end{gathered}
$$




$$
\begin{array}{r}
\sum_{e=1}^{N_{E}} x_{e} V_{e}-V^{*} \leq 0, \quad\left(V^{*}=\alpha V_{0}\right), \\
\text { where }: x_{e}=x_{\min } \text { or } 1, \quad\left(e=1, \ldots, N_{E}\right) .
\end{array}
$$

where $C_{d}$ denotes the dynamic-compliance, Uimplies the displacement amplitude vector of the steady-state response, $\mathbf{K}_{d} \equiv \mathbf{K}-\omega_{p}^{2} \mathbf{M}$ is defined as the dynamic stiffness matrix, where $\mathbf{U}$ and $\mathbf{K}_{d}$ can be acquired by Eq. (18) that shows the steady-state response formulation of the structure. $\mathbf{F}$ expresses the load vector of the external loading. The external loading vector $\mathbf{f}(t)$ with the given excitation frequency $\omega_{p}$ can be expressed as $\mathbf{f}(t)=\mathbf{F} e^{j \omega t}$ and the displacement response can be treated as $\mathbf{u}(t)=\mathbf{U} e^{j \omega t}$. The symbols $\mathbf{K}$ and $\mathbf{M}$ in Eq. (18) represent the global structural stiffness and mass matrices, respectively. The symbol $x_{e}$ in Eq. (19) is the design variable corresponding to element $e$. The parameter $\alpha$ in Eq. (19) shows the volume fraction of the available material, which is given by $V^{*} / V_{0} . V_{0}$ is the volume of the design domain and $V^{*}$ shows the desirable volume which is predefined. In Eq. (20), $x_{e}=x_{\min }$ and $x_{e}=1$ denote the void elements and solid elements. To avoid the singularity of the global dynamic stiffness matrix, a small value, e.g. 0.001 , is used. The symbol $N_{E}$ represents the total number of finite elements.

Sensitivity analysis

The sensitivity of the objective function $O b j=C_{d}$ in Eq. (17) with respect to the design variables $x_{e}$ is given by

$$
\frac{\partial C_{d}}{\partial x_{e}}=\frac{\partial \mathbf{F}^{T}}{\partial x_{e}} \mathbf{U}+\mathbf{F}^{T} \frac{\partial \mathbf{U}}{\partial x_{e}}
$$

where $\frac{\partial \mathbf{F}^{T}}{\partial x_{e}}$ denotes the sensitivity of external excitation respect to the design 
variable $x_{e}$, and the $\frac{\partial \mathbf{F}^{T}}{\partial x_{e}}=0$ holds when the external excitation is independent of the design variable $x_{e}$. Otherwise it can be handled using the method described by Hammer and Olhoff $[80,81] . \frac{\partial \mathbf{U}}{\partial x_{e}}$ means the derivative of amplitude of steady state response, and it is given by

$$
\left(\mathbf{K}-\omega_{p}^{2} \mathbf{M}\right) \frac{\partial \mathbf{U}}{\partial x_{e}}=f \equiv \frac{\partial \mathbf{F}}{\partial x_{e}}-\left(\frac{\partial \mathbf{K}}{\partial x_{e}}-\omega_{p}^{2} \frac{\partial \mathbf{M}}{\partial x_{e}}\right) \mathbf{U}
$$

where the vector $f \equiv \frac{\partial \mathbf{F}}{\partial x_{e}}-\left(\frac{\partial \mathbf{K}}{\partial x_{e}}-\omega_{p}^{2} \frac{\partial \mathbf{M}}{\partial x_{e}}\right) \mathbf{U}$ is known as the pseudo load. To solve Eq.(21), the adjoint method[82] is adopted. In bi-directional evolutionary structural optimization (BESO) method [19], as $x_{\min }$ tends to 0 (and $\mathrm{p}>1$ ), the sensitivity number $\alpha_{e}$ for the $e$ th element can be expressed by

$$
\alpha_{e}=\frac{\partial C_{d}}{\partial x_{e}}= \begin{cases}2 \mathbf{U}_{e}^{T} \frac{\partial \mathbf{F}_{e}}{\partial x_{e}}-\mathbf{U}_{e}^{\mathrm{T}}\left(p \mathbf{K}_{e}^{0}-\omega_{p}^{2} \mathbf{M}_{e}^{0}\right) \mathbf{U}_{e} & \text { when } x=1 \\ 2 \mathbf{U}_{e}^{T} \frac{\partial \mathbf{F}_{e}}{\partial x_{e}}+\mathbf{U}_{e}^{\mathrm{T}}\left(\omega_{p}^{2} \mathbf{M}_{e}^{0}\right) \mathbf{U}_{e} & \text { when } x=x_{\min }\end{cases}
$$

where $\mathbf{U}_{e}$ denotes the displacement response amplitude of element $e$.

When the external load $\mathbf{F}$ is independent of the design variable $x_{e}$, Eq. (23) can be simplified as

$$
\alpha_{e}=\frac{\partial C_{d}}{\partial x_{e}}= \begin{cases}-\mathbf{U}_{e}^{\mathrm{T}}\left(p \mathbf{K}_{e}^{0}-\omega_{p}^{2} \mathbf{M}_{e}^{0}\right) \mathbf{U}_{e} & \text { when } x=1 \\ \mathbf{U}_{e}^{\mathrm{T}}\left(\omega_{p}^{2} \mathbf{M}_{e}^{0}\right) \mathbf{U}_{e} & \text { when } x=x_{\min }\end{cases}
$$

\section{Dynamic optimization under multi-frequency (frequency band) excitation}

While the value of the external excitation of the structure is in a frequency range, such as $\left[\omega_{1}, \omega_{2}\right]$, a multi-frequency optimization problem is need to be considered. 
The commonly used method is to take the sub-objective function at each frequency or to separate the frequency band into discrete multiple sub-objective functions, and then we consider a weighted sum of the form as a new objective function to optimize, which can be described as follows

$$
\Pi=\int_{\omega_{1}}^{\omega_{2}} k_{c}(\omega) \Pi(\omega) d \omega
$$

where $k_{c}(\omega)$ denotes the weight coefficient of objective frequency $\omega, \Pi(\omega)$ represents the objective function corresponding to frequency $\omega$, and $\Pi$ represents the objective function of multi-frequency optimization. Similarly, in the multi-objective optimization problem, the sensitivity of multi-frequency optimization can be expressed as

$$
\begin{aligned}
& \Psi=\int_{\omega_{1}}^{\omega_{2}} k_{d}(\omega) \Psi(\omega) d \omega \\
& k_{d}(\omega)=k_{c}(\omega) k_{s}(\omega)
\end{aligned}
$$

where $\Psi(\omega)$ implies the sensitivity corresponding to frequency $\omega$, and $\Psi$ represents the sensitivity of multi-frequency optimization. $k_{d}(\omega)$ is the weight coefficient of objective frequency $\omega$, which is expressed in Eq. (27). $k_{s}(\omega)$ represents the sensitivity coefficient, which is used to correct the value of the sub-sensitivity of $\omega$. If the first sensitivity coefficient is defined as a constant, the sensitivity coefficient of the $i$ th frequency can be computed as follows:

$$
k_{s}\left(\omega_{i}\right)=\frac{\sum_{e=1}^{N_{E}} \Psi_{e}\left(\omega_{1}\right)}{\sum_{e=1}^{N_{E}} \Psi_{e}\left(\omega_{i}\right)} k_{s}\left(\omega_{1}\right) \quad e=1,2, \ldots \ldots, N_{E}
$$




\subsection{Eigenvalue topology optimization}

As the linear elastic structure of forced vibration, when the external frequencies are close to the eigenvalue of the structure, the dramatic amplification of the structural amplitude will appear. This is the occurrence of the so-called resonance phenomenon. Avoidance of resonance is one of the important objectives of structural dynamic performance design, which can be achieved by keeping the eigen-frequency of the structure away from the frequency of the external excitation. For structural optimization design, it is a feasible idea to change the eigenvalue of the structure by optimizing the mass and stiffness of the structure. In the topology optimization, this idea has been developed for structural eigenvalue optimization $[9,20,73]$. This method is presented below.

\section{Problem statement}

For a solid-void design, the eigenvalue optimization can be stated as

$$
\begin{gathered}
\max _{\rho_{e}, e=1, \ldots, N_{E}} \lambda_{o b j} \\
\text { Subject to: }\left(\mathbf{K}-\lambda_{o b j} \mathbf{M}\right) \boldsymbol{\varphi}_{o b j}=0 \\
\sum_{e=1}^{N_{E}} x_{e} V_{e}-V^{*} \leq 0, \quad\left(V^{*}=\alpha V_{0}\right), \\
\text { where : } x_{e}=x_{\min } \text { or } 1, \quad\left(e=1, \ldots, N_{E}\right) .
\end{gathered}
$$

where $\mathbf{K}$ is the global stiffness matrix, $\mathbf{M}$ is the global mass matrix. $\lambda_{o b j}$ denotes the objective eigenvalue of the structure and $\boldsymbol{\varphi}_{\text {obj }}$ represents the eigenvector corresponding to eigenvalue $\lambda_{o b j} . \lambda_{o b j}$ and $\boldsymbol{\varphi}_{o b j}$ are related to each other with the following Rayleigh quotient: 


$$
\lambda_{o b j}=\frac{\boldsymbol{\varphi}_{o b j}^{T} \mathbf{K} \varphi_{o b j}}{\boldsymbol{\varphi}_{o b j}^{T} \mathbf{M} \varphi_{o b j}}
$$

Sensitivity analysis

From Eq. (33), the sensitivity of the objective function $\lambda_{o b j}$ can be expressed as

$$
\frac{\partial \lambda_{o b j}}{\partial x_{e}}=\boldsymbol{\varphi}_{o b j}^{T}\left(\frac{\partial \mathbf{K}}{\partial x_{e}}-\lambda_{o b j} \frac{\partial \mathbf{M}}{\partial x_{e}}\right) \boldsymbol{\varphi}_{o b j}
$$

$\frac{\partial \mathbf{K}}{\partial x_{e}}$ and $\frac{\partial \mathbf{M}}{\partial x_{e}}$ can be directly obtained from Eq. (16). The sensitivity number for solid and soft elements can be expressed as

$$
\alpha_{e}=\frac{\partial \lambda_{o b j}}{\partial x_{e}}= \begin{cases}\boldsymbol{\varphi}_{o b j}^{T}\left(\frac{1-x_{\min }}{1-x_{\min }^{p}} p \mathbf{K}_{e}^{0}-\lambda_{o b j} \mathbf{M}_{e}^{0}\right) \boldsymbol{\varphi}_{o b j} & \text { when } x=1 \\ \boldsymbol{\varphi}_{o b j}^{T}\left(\frac{x_{\min }^{p-1}-x_{\min }^{p}}{1-x_{\min }^{p}} p \mathbf{K}_{e}^{0}-\lambda_{o b j} \mathbf{M}_{e}^{0}\right) \boldsymbol{\varphi}_{o b j} & \text { when } x=x_{\min }\end{cases}
$$

When $x_{\text {min }}$ tends to 0 (and $p>1$ ), the sensitivity numbers can be simplified as

$$
\alpha_{e}=\frac{\partial \lambda_{o b j}}{\partial x_{e}}= \begin{cases}\boldsymbol{\varphi}_{o b j}^{T}\left(p \mathbf{K}_{e}^{0}-\lambda_{o b j} \mathbf{M}_{e}^{0}\right) \boldsymbol{\varphi}_{o b j} & \text { when } x=1 \\ \boldsymbol{\varphi}_{o b j}^{T}\left(-\lambda_{o b j} \mathbf{M}_{e}^{0}\right) \boldsymbol{\varphi}_{o b j} & \text { when } x=x_{\min }\end{cases}
$$

\section{Robust dynamic property optimization under uncertainty}

\subsection{Dynamic-compliance topology optimization formulation}

The robust topology optimization model for minimizing the maximum dynamic-compliance under uncertainties can be derived from Eqs. (17) - (20) as follows:

$$
\begin{aligned}
\min _{\rho_{e}, e=1, \ldots, N_{E}} C_{d \max } & =\mathbf{F}^{T} \mathbf{U} \\
\text { Subject to: } C_{d \max } & =\mathrm{E}\left(\mathrm{C}_{d}\right)_{\max }+\kappa \mathrm{SD}\left(\mathrm{C}_{d}\right)_{\max } \\
\mathbf{K}_{\mathrm{d}} \mathbf{U} & \equiv\left(\mathbf{K}-\omega_{p}^{2} \mathbf{M}\right) \mathbf{U}=\mathbf{F}
\end{aligned}
$$




$$
\sum_{e=1}^{N_{E}} x_{e} V_{e}-V^{*} \leq 0, \quad\left(V^{*}=\alpha V_{0}\right),
$$

where : $x_{e}=x_{\min }$ or $1, \quad\left(e=1, \ldots, N_{E}\right)$.

where $\kappa$ is a predefined parameter. Combined with Eqs. (8) and (9), the maximum values of expectation $\mathrm{E}\left(C_{d}\right)_{\max }$ and standard variance $\mathrm{SD}\left(C_{d}\right)_{\max }$ are given as follows:

$$
\begin{aligned}
& \mathrm{E}\left(C_{d}\right)_{\max }=\frac{1}{2}\left(\mathbf{F}^{T} \mathbf{U}_{0}+\sum_{z} \sum_{y} \mathbf{F}^{T} \mathbf{U}_{1, z} \frac{\partial \mu\left(r_{z}(\mathbf{i})\right)}{\partial i_{y}^{I}} \Delta i_{y}\right) \\
& \mathrm{SD}\left(C_{d}\right)_{\max }=\frac{1}{2} \sqrt{\sum_{z}\left(\mathbf{F}^{T} \mathbf{U}_{2, x} \sigma\left(r_{x}\left(\mathbf{i}^{m}\right)\right)+\sum_{y}\left(\mathbf{F}^{T} \mathbf{U}_{3, x y} \sigma\left(r_{x}\left(\mathbf{i}^{m}\right)\right) \Delta i_{y}+\mathbf{F}^{T} \mathbf{U}_{2, x} \frac{\partial \sigma\left(r_{x}\left(\mathbf{i}^{m}\right)\right)}{\partial i_{l}} \Delta i_{y}\right)\right)^{2}}
\end{aligned}
$$

When the structures are under the external dynamic excitation, the equilibrium equation can be written as

$$
\mathbf{K}_{d}(\mathbf{r}(\mathbf{i})) \mathbf{U}(\mathbf{r}(\mathbf{i}))=\mathbf{F}(\mathbf{r}(\mathbf{i}))
$$

where $\mathbf{K}_{d}(\mathbf{r}(\mathbf{i})), \mathbf{U}(\mathbf{r}(\mathbf{i}))$ and $\mathbf{F}(\mathbf{r}(\mathbf{i}))$ represent the so-called dynamic-stiffness matrix, displacement vector and external dynamic load vector with interval random parameters, respectively. Substituting $\mu\left(\mathbf{r}\left(\mathbf{i}^{\mathrm{m}}\right)\right)$ into Eq. (40), we can obtain

$$
\mathbf{K}_{d}\left(\mu\left(\mathbf{r}\left(\mathbf{i}^{m}\right)\right)\right) \mathbf{U}\left(\mu\left(\mathbf{r}\left(\mathbf{i}^{m}\right)\right)\right)=\mathbf{F}\left(\mu\left(\mathbf{r}\left(\mathbf{i}^{m}\right)\right)\right)
$$

To solve Eqs. (10) -(13), combined with Eq.(41), the displacement vector $\mathbf{U}$ and the derivative of $\mathbf{U}$ with respect to the related interval random variables can be expressed as

$$
\begin{aligned}
& \mathbf{U}_{0}=\mathbf{K}_{\mathbf{d}}\left(\mu\left(\mathbf{r}\left(\mathbf{i}^{m}\right)\right)\right)^{-1} \mathbf{F}\left(\mu\left(\mathbf{r}\left(\mathbf{i}^{m}\right)\right)\right) \\
& \mathbf{U}_{1, z}=\mathbf{K}_{\mathbf{d}}\left(\mu\left(\mathbf{r}\left(\mathbf{i}^{m}\right)\right)\right)^{-1} \frac{\partial \mathbf{F}\left(\mu\left(\mathbf{r}\left(\mathbf{i}^{m}\right)\right)\right)}{\partial\left(\mu\left(r_{z}(\mathbf{i})\right)\right)}-\mathbf{K}_{\mathbf{d}}\left(\mu\left(\mathbf{r}\left(\mathbf{i}^{m}\right)\right)\right)^{-1} \frac{\partial \mathbf{K}_{\mathbf{d}}\left(\mu\left(\mathbf{r}\left(\mathbf{i}^{m}\right)\right)\right)}{\partial\left(\mu\left(r_{z}(\mathbf{i})\right)\right)} \mathbf{U}\left(\mu\left(\mathbf{r}\left(\mathbf{i}^{m}\right)\right)\right)
\end{aligned}
$$




$$
\begin{aligned}
& \mathbf{U}_{2, z}=\mathbf{U}_{1, z} \frac{\partial\left(\mu\left(r_{z}(\mathbf{i})\right)\right)}{\partial r_{z}} \\
& \mathbf{U}_{3, z y}=\mathbf{K}_{\mathbf{d}}\left(\mu\left(\mathbf{r}\left(\mathbf{i}^{m}\right)\right)\right)^{-1} \frac{\partial^{2} \mathbf{F}\left(\mu\left(\mathbf{r}\left(\mathbf{i}^{m}\right)\right)\right)}{\partial r_{z} \partial i_{y}}-\mathbf{K}_{\mathbf{d}}\left(\mu\left(\mathbf{r}\left(\mathbf{i}^{m}\right)\right)\right)^{-1} \frac{\partial^{2} \mathbf{K}_{\mathbf{d}}\left(\mu\left(\mathbf{r}\left(\mathbf{i}^{m}\right)\right)\right)}{\partial r_{z} \partial i_{y}} \mathbf{U}\left(\mu\left(\mathbf{r}\left(\mathbf{i}^{m}\right)\right)\right)
\end{aligned}
$$

\subsection{Sensitivity of dynamic-compliance robust optimization}

The sensitivity of dynamic-compliance robust optimization can be expressed as

$$
\begin{aligned}
& \frac{\partial \mathrm{E}\left(C_{d}\right)_{\max }}{\partial x_{e}}=\frac{1}{2}\left(\frac{\partial \mathbf{F}^{T} \mathbf{U}_{0}}{\partial x_{e}}+\sum_{y} \frac{\partial \mathbf{F}^{T} \mathbf{U}_{1, z}}{\partial x_{e}} \frac{\partial \mu\left(r_{z}(\mathbf{i})\right)}{\partial i_{y}^{I}} \Delta i_{y}\right) \\
& \sum_{z}\left(\mathbf{F}^{T} \mathbf{U}_{2, z} \sigma\left(r_{z}\left(\mathbf{i}^{m}\right)\right)+\sum_{y}\left(\mathbf{F}^{T} \mathbf{U}_{3, z y} \sigma\left(r_{z}\left(\mathbf{i}^{m}\right)\right) \Delta i_{y}+\mathbf{F}^{T} \mathbf{U}_{2, z} \frac{\partial \sigma\left(r_{z}\left(\mathbf{i}^{m}\right)\right)}{\partial i_{y}} \Delta i_{y}\right)\right) \\
& \frac{\partial \operatorname{SD}\left(C_{d}\right)_{\max }}{\partial x_{e}}=\frac{1}{2} \frac{\left(\frac{\partial \mathbf{F}^{T} \mathbf{U}_{2, z}}{\partial x_{e}} \sigma\left(r_{z}\left(\mathbf{i}^{m}\right)\right)+\sum_{y}\left(\frac{\partial \mathbf{F}^{T} \mathbf{U}_{3, z y}}{\partial x_{e}} \sigma\left(r_{z}\left(\mathbf{i}^{m}\right)\right) \Delta i_{y}+\frac{\partial \mathbf{F}^{T} \mathbf{U}_{2, z}}{\partial x_{e}} \frac{\partial \sigma\left(r_{z}\left(\mathbf{i}^{m}\right)\right)}{\partial i_{y}} \Delta i_{y}\right)\right)}{\sqrt{\sum_{z}\left(\mathbf{F}^{T} \mathbf{U}_{2, z} \sigma\left(r_{z}\left(\mathbf{i}^{m}\right)\right)+\sum_{y}\left(\mathbf{F}^{T} \mathbf{U}_{3, z y} \sigma\left(r_{z}\left(\mathbf{i}^{m}\right)\right) \Delta i_{y}+\mathbf{F}^{T} \mathbf{U}_{2, z} \frac{\partial \sigma\left(r_{z}\left(\mathbf{i}^{m}\right)\right)}{\partial i_{y}} \Delta i_{y}\right)\right)^{2}}}
\end{aligned}
$$

where $\frac{\partial \mathbf{F}^{T} \mathbf{U}_{0}}{\partial x_{e}} 、 \frac{\mathbf{F}^{T} \mathbf{U}_{1, x}}{\partial x_{e}} 、 \frac{\partial \mathbf{F}^{T} \mathbf{U}_{2, z}}{\partial x_{e}} 、 \frac{\partial \mathbf{F}^{T} \mathbf{U}_{3, z y}}{\partial x_{e}}$ can be obtained as

$$
\begin{aligned}
& \frac{\partial \mathbf{F}^{T} \mathbf{U}_{0}}{\partial x_{e}}=\mathbf{U}_{0}^{T} \frac{\partial \mathbf{K}_{\mathbf{d}}\left(\mu\left(\mathbf{r}\left(\mathbf{i}^{m}\right)\right)\right)}{\partial x_{e}} \mathbf{U}_{0} \\
& \frac{\partial \mathbf{F}^{T} \mathbf{U}_{1, z}}{\partial x_{e}}=-\mathbf{U}_{0}^{T} \frac{\partial \mathbf{K}_{\mathbf{d}}\left(\mu\left(\mathbf{r}\left(\mathbf{i}^{m}\right)\right)\right)}{\partial x_{e}} \mathbf{U}_{1, z}-\mathbf{U}_{0}^{T} \frac{\partial^{2} \mathbf{K}_{\mathbf{d}}\left(\mu\left(\mathbf{r}\left(\mathbf{i}^{m}\right)\right)\right)}{\partial x_{e} \partial\left(\mu\left(r_{z}(i)\right)\right)} \mathbf{U}_{0} \\
& \frac{\partial \mathbf{F}^{T} \mathbf{U}_{2, \mathrm{z}}}{\partial x_{e}}=\frac{\partial \mathbf{F}^{T} \mathbf{U}_{1, z} \frac{\partial\left(\mu\left(r_{z}(\mathbf{i})\right)\right)}{\partial x_{e}}}{\partial r_{z}} \\
& \frac{\partial \mathbf{F}^{T} \mathbf{U}_{3, z y}}{\partial x_{e}}=-\mathbf{U}_{0}^{T} \frac{\partial \mathbf{K}_{\mathbf{d}}\left(\mu\left(\mathbf{r}\left(\mathbf{i}^{m}\right)\right)\right)}{\partial x_{e}} \mathbf{U}_{3, z y}-\mathbf{U}_{0}^{T} \frac{\partial\left(\mu\left(r_{z}(\mathbf{i})\right)\right)^{2}}{\partial x_{e}} \mathbf{U}_{0}
\end{aligned}
$$

In Eq. (49) and Eq. (51), the terms $\frac{\mathbf{F}^{T} \mathbf{U}_{1, x}}{\partial x_{e}}$ and $\frac{\partial \mathbf{F}^{T} \mathbf{U}_{3, z y}}{\partial x_{e}}$ are caused by the 
uncertainties of the boundary conditions and material properties, respectively.

\subsection{Eigenvalue topology optimization formulation}

The robust eigenvalue optimization model aimed to maximize the minimum value of the target mode can be expressed as follows:

$$
\max _{\rho_{e}, e=1, \ldots, N_{E}}: \lambda_{o b j \min }
$$

Subject to: $\lambda_{\text {obj min }}=\mathrm{E}\left(\lambda_{\text {obj }}\right)_{\min }-\kappa \mathrm{SD}\left(\lambda_{o b j}\right)_{\max }$

$$
\begin{aligned}
& \left(\mathbf{K}-\lambda_{o b j} \mathbf{M}\right) \varphi_{o b j}=0 \\
& \sum_{e=1}^{N_{E}} x_{e} V_{e}-V^{*} \leq 0, \quad\left(V^{*}=\alpha V_{0}\right),
\end{aligned}
$$

where : $x_{e}=x_{\min }$ or $1, \quad\left(e=1, \ldots, N_{E}\right)$.

Considering the interval random parameters, $\mathrm{E}\left(\lambda_{o b j}\right)_{\min }$ and $\operatorname{SD}\left(\lambda_{\text {obj }}\right)_{\max }$ of the objective function can be expressed as

$$
\begin{aligned}
& \mathrm{E}\left(\lambda_{o b j}\right)_{\text {min }}=\lambda_{o b j}\left(\mu\left(\mathbf{r}\left(\mathbf{i}^{m}\right)\right)\right)-\sum_{z} \sum_{y} \frac{\partial \lambda_{o b j}\left(\mu\left(\mathbf{r}\left(\mathbf{i}^{m}\right)\right)\right)}{\partial \mu\left(r_{z}(\mathbf{i})\right)} \frac{\partial \mu\left(r_{z}(\mathbf{i})\right)}{\partial i_{y}^{I}} \Delta i_{y} \\
& =\lambda_{o b j 0}-\sum_{z} \sum_{y} \lambda_{o b j 1, z} \frac{\partial \mu\left(r_{z}(\mathbf{i})\right)}{\partial i_{y}^{I}} \Delta i_{y} \\
& \operatorname{SD}\left(\lambda_{o b j}\right)_{\max }=\sqrt{\left(\sum_{z} \frac{\partial \lambda_{o b j}\left(\mu\left(r\left(\mathbf{i}^{m}\right)\right)\right)}{\partial r_{z}} \sigma\left(r_{z}\left(\mathbf{i}^{m}\right)\right)+\sum_{y}\left(\begin{array}{l}
\frac{\partial^{2} \lambda_{o b j}\left(\mu\left(\mathbf{r}\left(\mathbf{i}^{m}\right)\right)\right)}{\partial r_{z} \partial i_{y}} \sigma\left(r_{z}\left(\mathbf{i}^{m}\right)\right) \Delta i_{y} \\
+\frac{\partial \lambda_{o b j}\left(\mu\left(\mathbf{r}\left(\mathbf{i}^{m}\right)\right)\right)}{\partial r_{z}} \frac{\partial \sigma\left(r_{z}\left(\mathbf{i}^{m}\right)\right)}{\partial i_{y}} \Delta i_{y}
\end{array}\right)\right)^{2}} \\
& =\sqrt{\sum_{z}\left(\lambda_{o b j, 2, z} \sigma\left(r_{z}\left(\mathbf{i}^{m}\right)\right)+\sum_{y}\left(\lambda_{o b j, z y} \sigma\left(r_{z}\left(\mathbf{i}^{m}\right)\right) \Delta i_{y}+\lambda_{o b j, z,} \frac{\partial \sigma\left(r_{z}\left(\mathbf{i}^{m}\right)\right)}{\partial i_{y}} \Delta i_{y}\right)\right)^{2}}
\end{aligned}
$$

Combined with Eq.(33), $\lambda_{o b j 0} 、 \lambda_{o b j 1, z} 、 \lambda_{o b j 2, \mathrm{z}} 、 \lambda_{o b j 3, z y}$ can be obtained as

$$
\lambda_{o b j 0}=\frac{\boldsymbol{\varphi}_{o b j}^{T} \mathbf{K}\left(\mu\left(\mathbf{r}\left(\mathbf{i}^{m}\right)\right)\right) \boldsymbol{\varphi}_{o b j}}{\boldsymbol{\varphi}_{o b j}^{T} \mathbf{M}\left(\mu\left(\mathbf{r}\left(\mathbf{i}^{m}\right)\right)\right) \boldsymbol{\varphi}_{o b j}}
$$




$$
\begin{aligned}
& \lambda_{o b j, z}=\boldsymbol{\varphi}_{o b j}^{T}\left(\frac{\partial \mathbf{K}\left(\mu\left(\mathbf{r}\left(\mathbf{i}^{m}\right)\right)\right)}{\partial \mu\left(r_{z}(\mathbf{i})\right)}-\lambda_{o b j} \frac{\partial \mathbf{M}\left(\mu\left(\mathbf{r}\left(\mathbf{i}^{m}\right)\right)\right)}{\partial \mu\left(r_{z}(\mathbf{i})\right)}\right) \boldsymbol{\varphi}_{o b j} \\
& \lambda_{o b j, \mathrm{z}}=\lambda_{o b j \mathrm{j}, \mathrm{z}} \frac{\partial \mu\left(r_{z}(\mathbf{i})\right)}{\partial r_{z}} \\
& \lambda_{o b j 3, z y}=\boldsymbol{\varphi}_{o b j}^{T}\left(\frac{\partial^{2} \mathbf{K}\left(\mu\left(\mathbf{r}\left(\mathbf{i}^{m}\right)\right)\right)}{\partial r_{z} \partial i_{y}}-\lambda_{o b j} \frac{\partial \mathbf{M}\left(\mu\left(\mathbf{r}\left(\mathbf{i}^{m}\right)\right)\right)}{\partial r_{z} \partial i_{y}}\right) \boldsymbol{\varphi}_{o b j}
\end{aligned}
$$

\subsection{Sensitivity of eigenvalue}

The sensitivity of robust eigenvalue optimization can be expressed as:

$$
\begin{aligned}
& \frac{\partial \mathrm{E}\left(\lambda_{o b j}\right)_{\min }}{\partial x_{e}}=\frac{\partial \lambda_{o b j 0}}{\partial x_{e}}-\sum_{z} \sum_{y}\left(\frac{\partial \lambda_{o b j 1, z}}{\partial x_{e}} \frac{\partial \mu\left(r_{z}(\mathbf{i})\right)}{\partial i_{y}^{I}} \Delta i_{y}\right)
\end{aligned}
$$

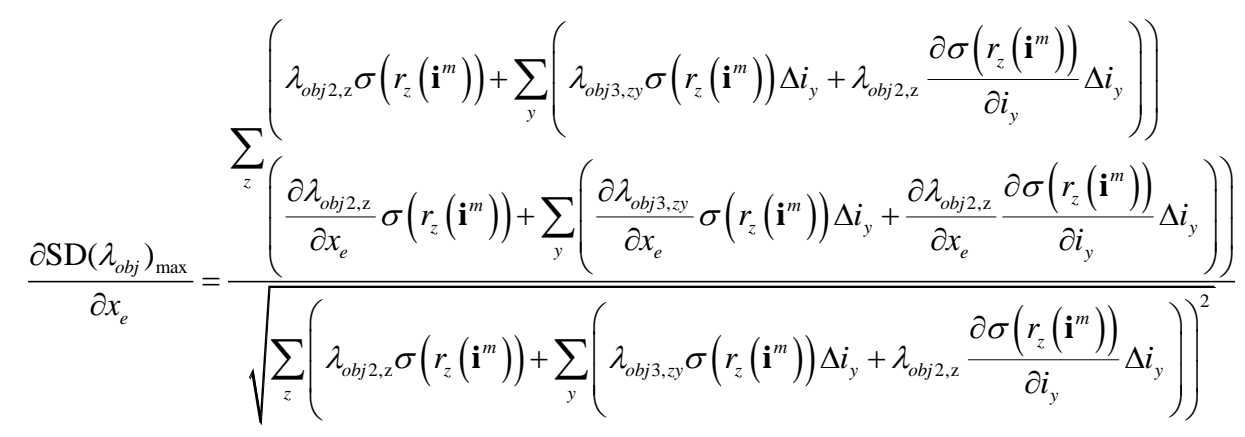

Combining with Eq.(34), $\frac{\partial \lambda_{o b j 0}}{\partial x_{e}} 、 \frac{\partial \lambda_{o b j 1, z}}{\partial x_{e}}, \frac{\partial \lambda_{o b j 2, z}}{\partial x_{e}}, \frac{\partial \lambda_{o b j 3, z y}}{\partial x_{e}}$ can be obtained as:

$$
\begin{aligned}
& \frac{\partial \lambda_{o b j}}{\partial x_{e}}=\boldsymbol{\varphi}_{o b j}^{T}\left(\frac{\partial \mathbf{K}\left(\mu\left(\mathbf{r}\left(\mathbf{i}^{m}\right)\right)\right)}{\partial x_{e}}-\lambda_{o b j} \frac{\partial \mathbf{M}\left(\mu\left(\mathbf{r}\left(\mathbf{i}^{m}\right)\right)\right)}{\partial x_{e}}\right) \boldsymbol{\varphi}_{o b j} \\
& \frac{\partial \lambda_{o b j 1, z}}{\partial x_{e}}=\boldsymbol{\varphi}_{o b j}^{T}\left(\frac{\partial \mathbf{K}\left(\mu\left(\mathbf{r}\left(\mathbf{i}^{m}\right)\right)\right)}{\partial \mu\left(r_{z}(\mathbf{i})\right)} / \partial x_{e}-\lambda_{o b j} \frac{\partial \mathbf{M}\left(\mu\left(\mathbf{r}\left(\mathbf{i}^{m}\right)\right)\right)}{\partial \mu\left(r_{z}(\mathbf{i})\right)} / \partial x_{e}\right) \boldsymbol{\varphi}_{o b j} \\
& \frac{\partial \lambda_{o b j 2, z}}{\partial x_{e}}=\frac{\partial \lambda_{o b j 1, z}}{\partial x_{e}} \frac{\partial \mu\left(r_{z}(\mathbf{i})\right)}{\partial r_{z}}
\end{aligned}
$$




$$
\frac{\partial \lambda_{o b j 3, z y}}{\partial x_{e}}=\boldsymbol{\varphi}_{o b j}^{T}\left(\frac{\partial^{2} \mathbf{K}\left(\mu\left(\mathbf{r}\left(\mathbf{i}^{m}\right)\right)\right)}{\partial r_{z} \partial i_{y}} / \partial x_{e}-\lambda_{o b j} \frac{\partial \mathbf{M}\left(\mu\left(\mathbf{r}\left(\mathbf{i}^{m}\right)\right)\right)}{\partial r_{z} \partial i_{y}} / \partial x_{e}\right) \boldsymbol{\varphi}_{o b j}
$$

\section{Numerical implementation}

\subsection{Filter scheme}

The filter schemes have been used by finite element method based topology optimization methods in order to avoid numerical instabilities [83, 84]. A spatial linear filter is used in this work to smooth the sensitivity numbers to prevent checker boards and mesh-dependence. The filtering procedure starts with distributing the elemental sensitivity numbers to the mesh nodes according to their nodal connectivity, which is expressed as follows

$$
\alpha_{i}=\frac{\sum_{j=1}^{K} w\left(r_{i j}\right) \alpha_{j}}{\sum_{j=1}^{K} w\left(r_{i j}\right)}
$$

where $K$ denotes the total number of nodes in sub-domain $\Omega_{i}$. The sub-domain $\Omega_{i}$ is generated by drawing a circle of radius $r_{\min }$, which is the center of the $i$ th element. $r_{i j}$ represents the distance between the center of element $i$ and element $j . \alpha_{j}$ is the sensitivity number of element $j . w\left(r_{i j}\right)$ is the linear weight factor defined as

$$
w\left(r_{i j}\right)= \begin{cases}r_{\min }-r_{i j} & \text { for } r_{i j}<r_{\min } \\ 0 & \text { for } r_{i j} \geq r_{\min }\end{cases}
$$

\subsection{Sensitivity history}

A history-averaging of the sensitivities is used to help stabilize the optimization process $[52,53]$. The smoothed sensitivity number from Eq. (24) and (36) is averaged here with their value of the previous iteration. 


$$
\alpha_{i}=\frac{\alpha_{i}^{k}+\alpha_{i}^{k-1}}{2}
$$

where $k$ represents the number of current iteration.

\subsection{Convergence criterion}

Once the predefined final volume is achieved, the optimization procedure continues until the objective function converges. The convergence check is expressed as follows:

$$
\text { error }=\frac{\left|\sum_{i=1}^{N} C_{k-i+1}-\sum_{i=1}^{N} C_{k-N-i+1}\right|}{\sum_{i=1}^{N} C_{k-i+1}} \leq \tau
$$

where $C_{k}$ represents the objective function value in the $k$ th iteration. $N$ is usually set to 5 , which means that the change of the objective function in the last 10 iterations is small enough. $\tau$ represents the tolerance of change.

\subsection{Summary of the numerical implementation}

The main step of the proposed robust topology optimization for dynamic problems with the interval random variables is given in Fig.1. 


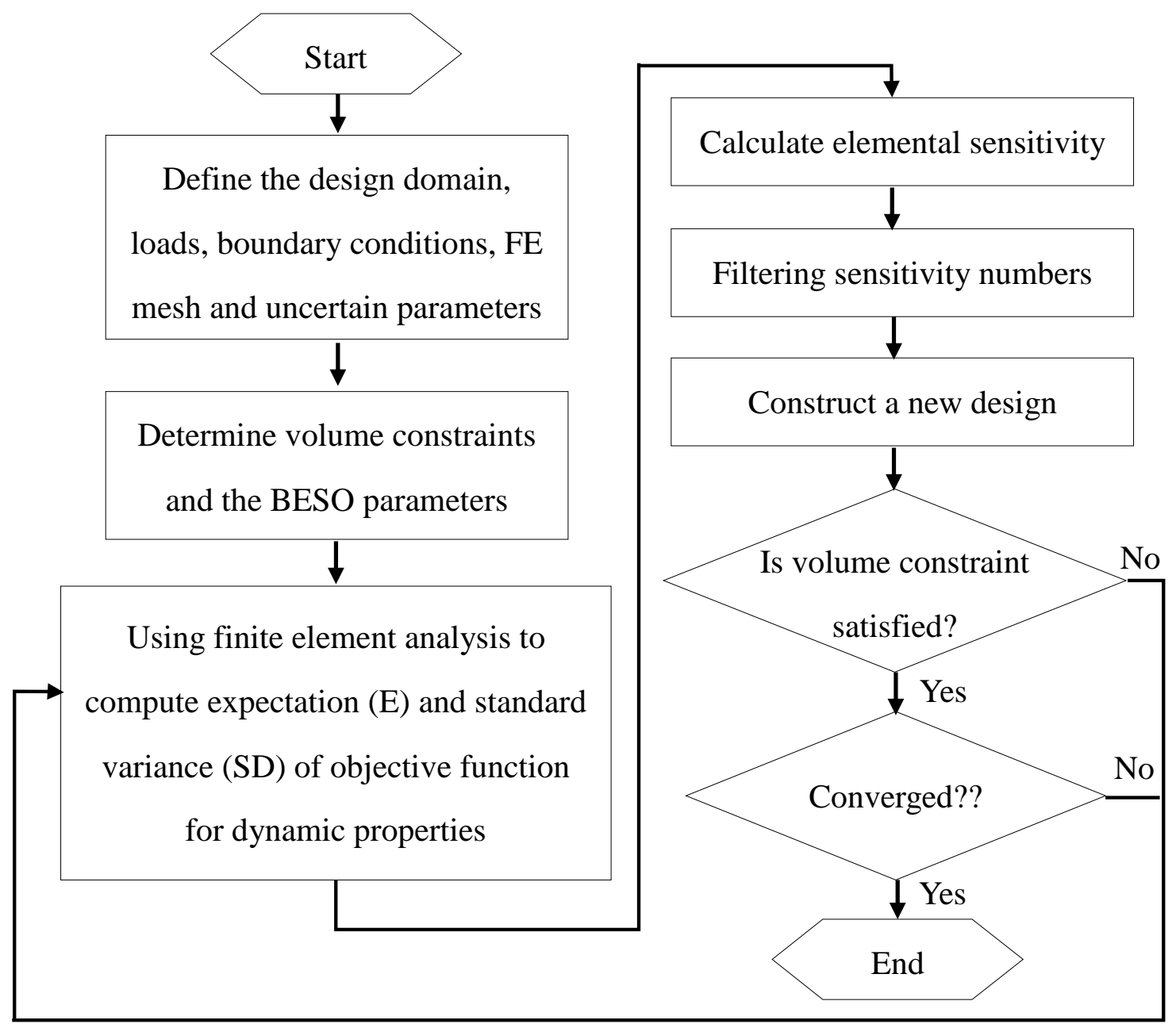

Fig.1 Main step of the solution algorithm

\section{Numerical examples}

In this section, three examples are presented. The first two examples are the robust dynamic-compliance optimization cases, and the last example is a robust eigenvalue optimization problem. It is assumed for all cases that the base deterministic material has Young's modulus $E=210 \mathrm{GPa}$, Poisson's ratio $v=0.3$ and density $\rho=7900 \mathrm{~g} / \mathrm{cm}^{3}$. And the design variable $x=1 \mathrm{~mm}$ or $0.01 \mathrm{~mm}$. The Young's modulus, density and Poisson's ratio of material, the design variable and the angle of external excitation are assumed to be interval random variables and the random parameters follow the normal distribution. The intervals of the expectation and 
standard variance of the uncertain parameters are given below:

$$
\begin{aligned}
& \mu(E)^{I}=[189,231] \mathrm{GPa}, \sigma(E)^{I}=[18.9,23.1] \mathrm{GPa} \\
& \mu(v)^{I}=[0.285,0.315], \sigma(v)^{I}=[0.01425,0.01575] \\
& \mu(\rho)^{I}=[7505,8205] \mathrm{g} / \mathrm{cm}^{3}, \sigma(\rho)^{I}=[750.5,820.5] \mathrm{g} / \mathrm{cm}^{3} \\
& \mu(x)^{I}=[0.95,1.05] \mathrm{mm}, \sigma(x)^{I}=[0.0475,0.0525] \mathrm{mm} \\
& \mu(\theta)^{I}=\left[0^{\circ}, 3^{\circ}\right], \sigma(\theta)^{I}=\left[0.27^{\circ}, 0.33^{\circ}\right]
\end{aligned}
$$

Where $\mu$ and $\sigma$ stand for the expectation and standard variance, respectively, and $E, v$, $\rho, x, \theta$ denote the Young's modulus, Poisson's ratio, density, the design variable and the angle of external excitation, respectively. The value of the predefined coefficient $\kappa$ is set to 1 in the numerical examples of this section.

Four-node quadrilateral elements are used. Several dynamic work conditions under hybrid interval random uncertain are discussed. In the BESO method, the evolutionary ratio is set to $2 \%$, and the filter radius is $20 \mathrm{~mm}$. The penalty exponent $p=3.0$ is used in calculating the sensitivity numbers.

6.1 Dynamic-compliance optimization with independent uncertainty in cantilever beam

The first example considers the dynamic-compliance topology optimization with interval random uncertainties of material properties, geometrical properties and boundary conditions in a long cantilever. These variables represent three main uncertainties in real engineering [85]. 


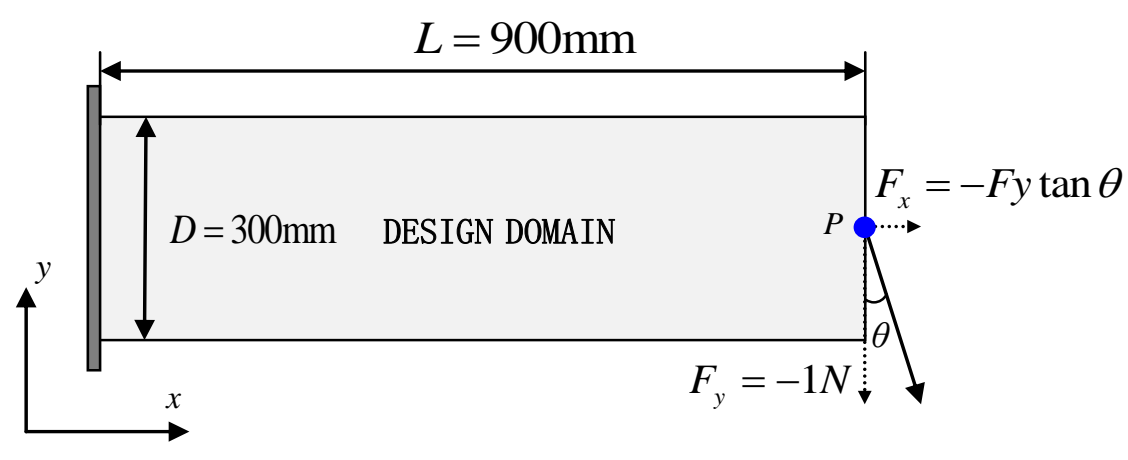

Fig. 2. Design domain of a cantilever beam

The design domain, boundary conditions and external load of the cantilever are shown in Fig.2. The left end of the cantilever is fixed, and an external excitation with frequency is loaded on the middle of the right end which is marked as $P$. The excitation on $y$ direction is deterministic, and it is interval random variable on $x$ direction which can be described as $F_{x}=-F_{y} \tan \theta$, where the symbol '-' contributes the direction. Due to the presence of the parameters defined before, the structural dynamic-compliance of the cantilever is an interval random parameter, which can be carried out with Eqs. (38) and (39), and the topology design can be formulated with the iteration of the sensitivity of dynamic-compliance. The design variable $x_{i}$ is set to be either 1 or $x_{\min }=0.001$ in this example and the volume fraction $V^{*}$ is set to $50 \%$

Fig.3 shows the dynamic-compliance topology design results for the cantilever under different external excitation frequency carried out by RTO with interval random parameters. Fig. 3(a-c), Fig. 3(d-f) and Fig. 3(g-i) present the optimal design under material, geometrical and boundary uncertainties, respectively. For the purpose of comparison, Fig. 3 (j-1) is employed to show the optimal topology design under deterministic parameters. 


\begin{tabular}{|c|c|c|c|}
\hline & $0 \mathrm{~Hz}$ (Static case) & $50 \mathrm{~Hz}$ & $100 \mathrm{~Hz}$ \\
\hline $\begin{array}{c}\text { Material } \\
\text { uncertainty }\end{array}$ & (a) & (b) & (c) \\
\hline $\begin{array}{c}\text { Geometrical } \\
\text { uncertainty }\end{array}$ & (d) & (e) & (f) \\
\hline $\begin{array}{l}\text { Boundary } \\
\text { condition } \\
\text { uncertainty }\end{array}$ & (g) & (h) & (i) \\
\hline $\begin{array}{l}\text { Deterministi } \\
\mathrm{c} \text { results }\end{array}$ & (j) & (k) & (1) \\
\hline
\end{tabular}

Fig.3 Optimal topology design under various uncertainties

It can be observed that, in Fig.3 (a-c), the optimal designs of the structure under material uncertainties are much different from the deterministic results. In addition, the optimal structures are various at different frequencies under the interval random uncertainties. Fig.3 (d-f) shows the optimal topology design considering geometrical uncertainties. In this condition, the optimal design is more similar to the deterministic design, because the perturbation of thickness is tiny and the contribution for the dynamic property is less than the material uncertainties do in the SIMP material model. Fig.3 (g-i) presents the results of robust topology optimization under boundary condition uncertainties (loading angle) in this example. The uncertain force of $x$-direction leads to the variation of the optimal design and as the changing of external 
loading frequency, the different topology design results presented. The numerical results presented in this section has clearly indicated that the topology optimization of structure for dynamic properties considering the interval random uncertainties is extremely different from that in static case. In addition, the uncertainty from different factors is crucial to determine the final layout of optimized structure.

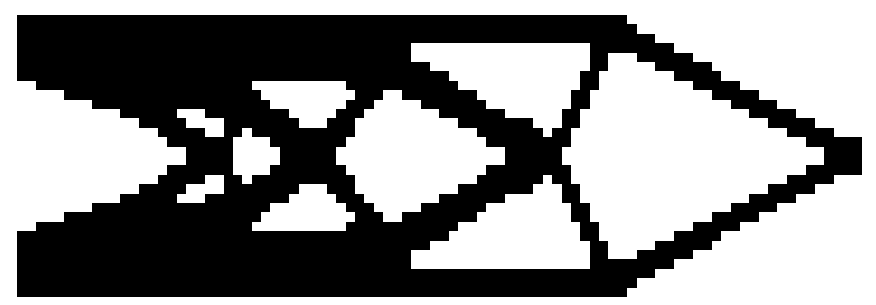

Fig. 4 Topology optimization result with frequency band excitation $(10-350 \mathrm{~Hz})$ under interval random uncertainties of material

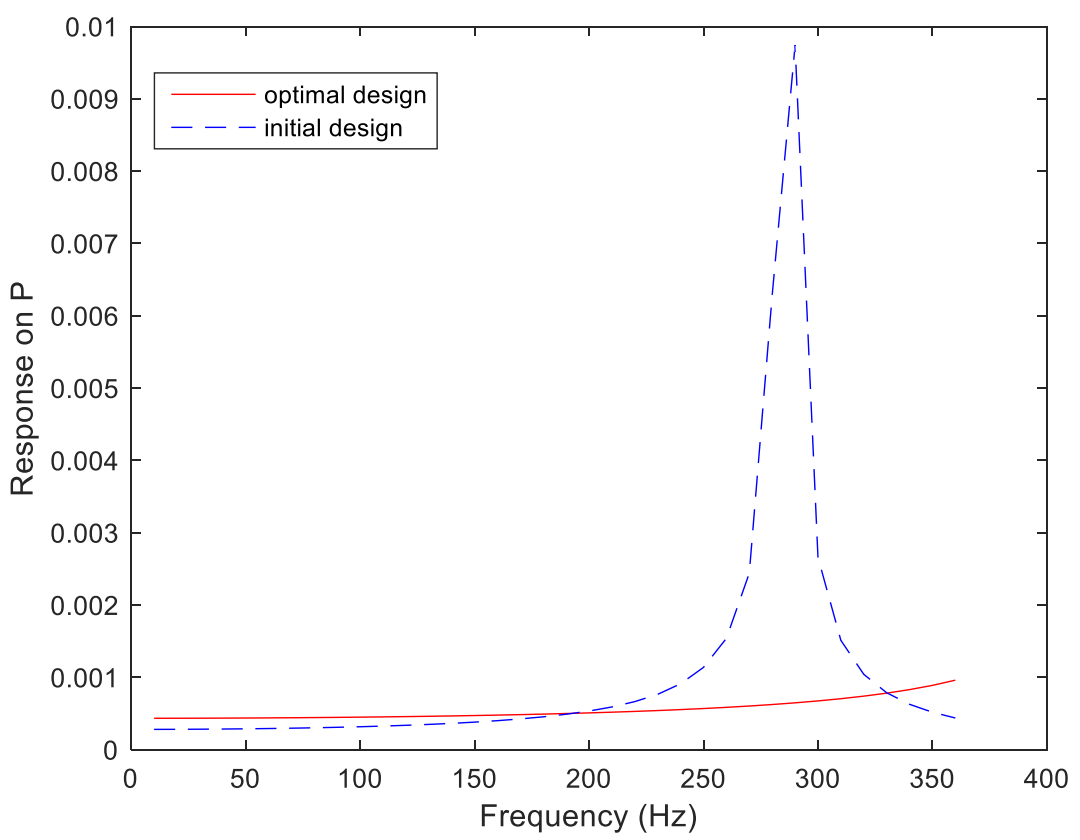

Fig. 5 Frequency response comparison between the initial and final optimized design on point $P$ considering interval random uncertainties of material

Fig. 4 shows the layout after the optimal topology design of the dynamic-compliance under frequency band excitations with interval random material uncertainty. The frequency band of external excitation is loaded from $10 \mathrm{~Hz}$ to $350 \mathrm{~Hz}$. 
That means the optimal topology design is considered as a multi-objective optimization problem. In such complex conditions, the optimal topology design presents a completely different form.

Fig. 5 compares the frequency response curve of point $\mathrm{P}$ from $10 \mathrm{~Hz}$ to $350 \mathrm{~Hz}$ between the optimized and original structures. The full line and dashed line represent the frequency response of optimal design and original design, respectively. From this Figure, it can be seen that when the initial design is under an external load with frequency from $200 \mathrm{~Hz}$ to $350 \mathrm{~Hz}$, the response at point $\mathrm{P}$ in the structure reaches a peak, which is inadvisable for structural dynamic performance. By adopting the RTO method for dynamic compliance under multi-frequency excitation from $10 \mathrm{~Hz}$ to $350 \mathrm{~Hz}$, it is found that the frequency response of the optimized structure shows a better dynamic performance. In addition, it is noticed that the response peak from $200 \mathrm{~Hz}$ to $350 \mathrm{~Hz}$ also disappears, which means that the structure is not under the severe vibration at the target frequency. In summary, the efficient algorithm developed in this work provides an excellent tool to optimize the structure and avoid the occurrence of resonance in the frequency band. This is extremely important in the design of continuum structure under the dynamic circumstances.

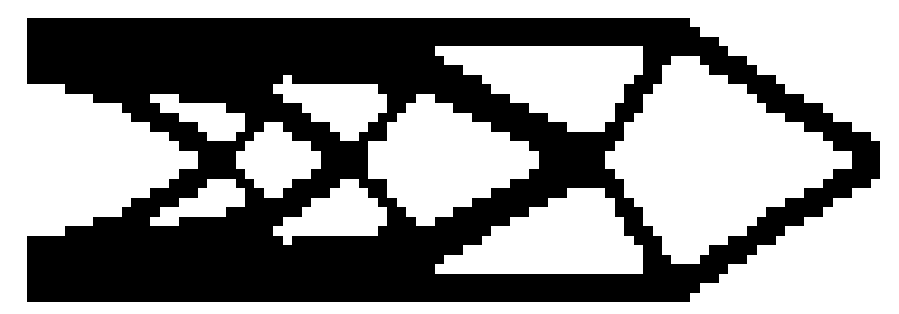

Fig.6 Topology design with a frequency band $(10-200 \mathrm{~Hz})$ under interval random variables of geometry. 
Fig. 6 shows the optimal robust topology design under geometrical uncertainties with a frequency band $(10-200 \mathrm{~Hz})$. It can be seen that even if the structure is under the influence of geometrical uncertainties which have low sensitivity, the final optimal topology design results are still different. This indicates that the dynamic problem has the characteristics of high sensitivity.

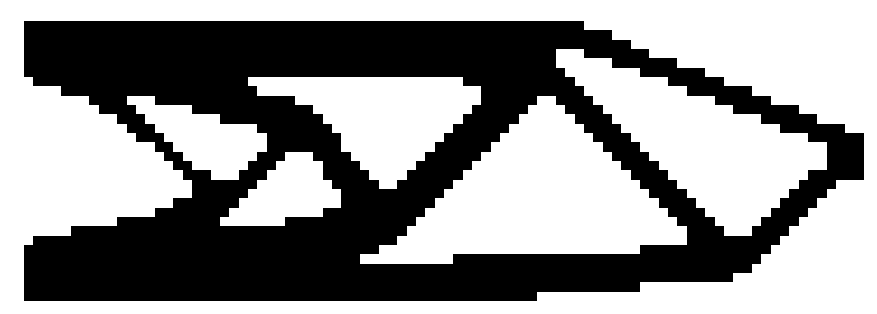

Fig.7A new topology design with a frequency band $(10-210 \mathrm{~Hz})$ excitation under interval random loading angle uncertainties

Fig. 7 presents the optimized dynamic-compliance topology design considering an interval random loading angle with a frequency band $(10-210 \mathrm{~Hz})$, which is different from the optimal design considering a single frequency. Because of the interval random load forced at $x$-direction, the dynamic-compliance becomes different. Considering the fact that there are a lot of loading frequencies in the practical condition, the powerful optimization model established in this paper can be effectively applied to the complicated engineering problems. 
6.2 Dynamic-compliance optimization with simultaneous uncertainty in a clamped-clamped beam

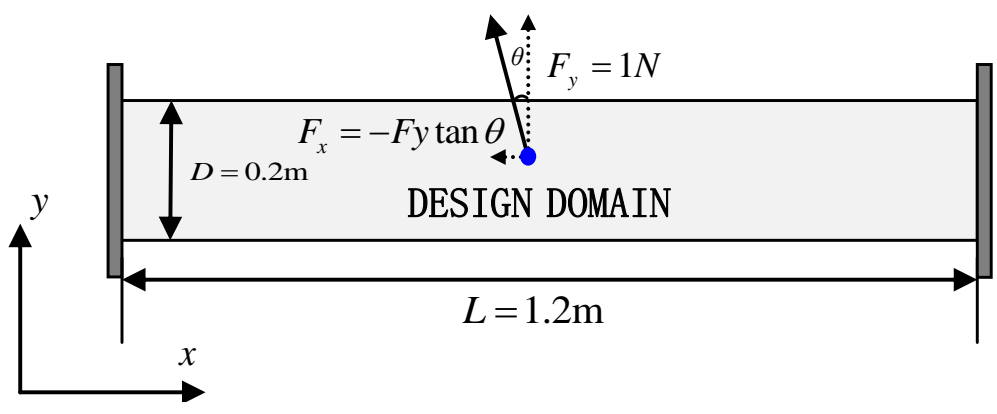

Fig.8Illustration of a clamped-clamped beam

Fig. 8 depicts a clamped-clamped beam of dimensions $1200 \mathrm{~mm} \times 200 \mathrm{~mm}$. The design domain is discretized with $120 \times 20$ four node quadrilateral element. The design variable $x_{i}$ is set to be either 1 or $x_{\min }=0.001$ in this example and the volume fraction $V^{*}$ is set to $50 \%$. The basic parameters of the material are the same as the previously described one, and these parameters are also uncertain.



Fig.9 Topology optimization with simultaneous interval random parameters. 




Fig. 10 Evolution history of the robust dynamic-compliance topology optimization (frequency is $50 \mathrm{~Hz}$ )

Fig.9 shows the topology optimization results with interval random uncertainties of material, geometrical and boundary condition, simultaneously for different frequency, and a typical evolution history (frequency is $50 \mathrm{~Hz}$ ), is shown in Fig. 10. For the purpose of comparison, the optimization results of the deterministic design are also shown in this figure. As outlined in Fig. 9, there is an obvious difference between the deterministic topology designs and the uncertain topology designs considering the interval random variables. It is well known that the simultaneous uncertainties from manufacturing, measurement and installation are unavoidable in real engineering problems. Therefore, it is very necessary to carry on the topology optimization for dynamic properties of structure with uncertain effects. The proposed algorithm developed in this work provides a powerful tool to improve the design of structure with the uncertainty. 




Fig.11: Design domain of a simply supported beam

In this example, the robust objective is to maximize the first bending natural eigenvalue of a beam-like 2D structure with simply supported ends shown in Fig. (11) for a prescribed volume fraction $V^{*}=50 \%$ of the design domain and the design variable $x_{i}$ is set to be either 1 or $x_{\min }=10^{-6}$ in this example [19]. The rectangular design domain of $1600 \mathrm{~mm} \times 200 \mathrm{~mm}$ is divided into $160 \times 20$ four node quadrilateral element. The material parameters are defined in Eq. (69).

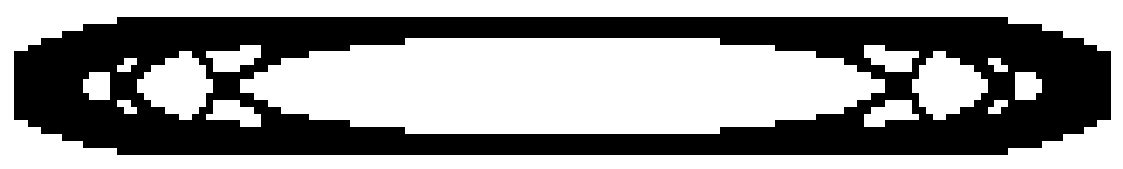

(a) material and geometrical uncertainty

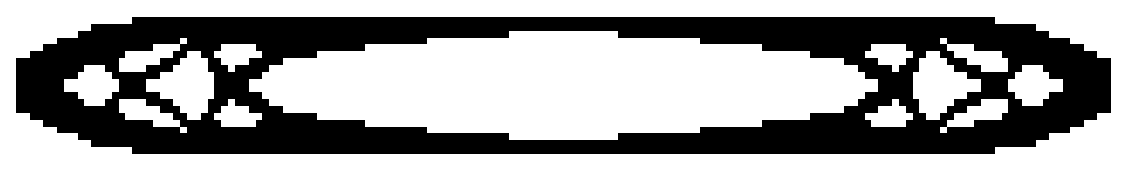

(b)material uncertainty



(b)geometrical uncertainty 




(d) deterministic model

Fig. 12 Results of first bending modal frequency topology optimization

Fig. 12 (a) shows the topology optimization results for the $1^{\text {st }}$ order of eigenvalue under simultaneous effects with material and geometrical uncertainty. In order to illustrate the individual effect of uncertainty factors, the optimized layouts for the $1^{\text {st }}$ order of eigenvalue are outlined in Figs. 12 (b) and (c) with material and geometrical uncertainty, respectively. The results of the deterministic topology optimization are shown in Fig. 12 (d) for comparison. It can be clearly observed from Fig. 12 that the uncertainties from material properties and geometry play a very important role to determine the final layouts. The slight variance of material properties and geometry may result in an obvious difference between the deterministic and uncertainty design. The efficient algorithm of dynamic topology optimization with consideration of uncertainty developed in this work has been demonstrated again by this example.

\section{Conclusions}

This paper deals with robust topology optimization (RTO) for dynamic properties considering hybrid uncertain parameters. The material, geometrical and boundary uncertainty are modeled with the interval random model. Two typical dynamic properties, including the frequency response and eigenvalue, of the continuum structure are chosen as the objective function. The expressions of the dynamic-compliance of frequency response and eigenvalue with interval random 
uncertainty and its sensitivities are derived.

With consideration of the hybrid interval random parameter, two numerical examples are presented to compare the robust dynamic-compliance topology optimization with deterministic topology optimization and there are great differences between the robust results with hybrid interval random uncertainty and deterministic parameters. Additionally, the dynamic-compliance topology design considering a frequency band excitation is also presented. It can be seen that the robust dynamic-compliance topology optimization method would reduce the frequency response of the structure efficiently, which is significant in practical engineering. And another numerical example is shown to present the robust eigenvalue topology optimization with hybrid interval random parameters. The examples distinctly show that the uncertainties of material, geometry and boundary condition have a considerable effect on the optimal topology design. This robust topology optimization method is very effective to design the structure under dynamic circumstances with hybrid interval random uncertainties. Furthermore, the efficient algorithm established in this work can be easily extended to multi-physics domains.

\section{Acknowledgements}

The project is supported by the Project funded by China Postdoctoral Science Foundation. The authors also wish to thank Research Project of the Science Fund of State Key Laboratory of Advanced Design and Manufacturing for Vehicle Body (Grant No. 51375001 and 31615002), Research Project of State Key Laboratory of Mechanical Systems and Vibration (MSV 201613 and MSV201711), and Open Foundation of the State Key Laboratory of Fluid Power and Mechatronic Systems GZK F-201601. 


\section{References}

[1] M.P. Bendsoe, O. Sigmund, Topology optimization: theory, methods, and applications, Springer Science \& Business Media2013.

[2] Y.M. Xie, G.P. Steven, A Simple Evolutionary Procedure for Structural Optimization, Comput Struct, 49 (1993) 885-896.

[3] M.Y. Wang, X. Wang, D. Guo, A level set method for structural topology optimization, Computer Methods in Applied Mechanics \& Engineering, 192 (2003) 227-246.

[4] Z.C. He, G.Y. Zhang, L. Deng, E. Li, G.R. Liu, Topology Optimization Using Node-Based Smoothed Finite Element Method, International Journal of Applied Mechanics, 07 (2015) 1550085.

[5] E. Li, C.C. Chang, Z.C. He, Z. Zhang, Q. Li, Smoothed finite element method for topology optimization involving incompressible materials, Engeering Optimization, (2016) 1-27.

[6] X. Huang, M. Xie, Evolutionary topology optimization of continuum structures: methods and applications, John Wiley \& Sons2010.

[7] M.P. Bendsøe, N. Kikuchi, Generating optimal topologies in structural design using a homogenization method, Computer Methods in Applied Mechanics \& Engineering, 71 (1988) 197-224.

[8] W.M. Vicente, Z.H. Zuo, R. Pavanello, T.K.L. Calixto, R. Picelli, Y.M. Xie, Concurrent topology optimization for minimizing frequency responses of two-level hierarchical structures, Computer Methods in Applied Mechanics \& Engineering, 301 (2016) 116-136.

[9] N. Olhoff, J. Du, On topological design optimization of structures against vibration and noise emission, Computational Aspects of Structural Acoustics and Vibration, (2009) 217-276.

[10] N. Olhoff, J. Du, Topology optimization of structures against vibration and noise, Proceedings of the 12th International Congress on Sound and Vibration, 2005.

[11] S. Min, S. Nishiwaki, N. Kikuchi, Unified topology design of static and vibrating structures using multiobjective optimization, Computers \& Structures, 75 (2000) 93-116.

[12] L. Shu, M.Y. Wang, Z. Fang, Z. Ma, P. Wei, Level set based structural topology optimization for minimizing frequency response, Journal of Sound \& Vibration, 330 (2011) 5820-5834.

[13] Z.D. Ma, N. Kikuchi, H.C. Cheng, Topological design for vibrating structures, Computer Methods in Applied Mechanics \& Engineering, 121 (1995) 259-280.

[14] J.-H. Zhu, W.-H. Zhang, L. Xia, Topology Optimization in Aircraft and Aerospace Structures Design, Archives of Computational Methods in Engineering, 23 (2016) 595-622.

[15] A.R. Díaaz, N. Kikuchi, Solutions to shape and topology eigenvalue optimization problems using a homogenization method, International Journal for Numerical Methods in Engineering, 35 (1992) 1487-1502.

[16] Z.D. Ma, N. Kikuchi, I. Hagiwara, Structural topology and shape optimization for 
a frequency response problem, Computational Mechanics, 13 (1993) 157-174.

[17] S. Min, N. Kikuchi, Y. Park, S. Kim, S. Chang, Optimal topology design of structures under dynamic loads, Structural optimization, 17 (1999) 208-218.

[18] C.S. Jog, TOPOLOGY DESIGN OF STRUCTURES SUBJECTED TO PERIODIC LOADING, Journal of Sound \& Vibration, 253 (2002) 687-709.

[19] J. Du, N. Olhoff, Topological design of freely vibrating continuum structures for maximum values of simple and multiple eigenfrequencies and frequency gaps, Structural \& Multidisciplinary Optimization, 34 (2007) 91-110.

[20] Y.M. Xie, G.P. Steven, Evolutionary structural optimization for dynamic problems, Computers \& Structures, 58 (1996) 1067-1073.

[21] X. Huang, Z.H. Zuo, Y.M. Xie, Evolutionary topological optimization of vibrating continuum structures for natural frequencies, Computers \& Structures, 88 (2010) 357-364.

[22] K. Yan, G. Cheng, B.P. Wang, Topology optimization of plate structures subject to initial excitations for minimum dynamic performance index, Structural and Multidisciplinary Optimization, 53 (2016) 623-633.

[23] S.R. Arwade, M. Grigoriu, Probabilistic Model for Polycrystalline Microstructures with Application to Intergranular Fracture, Journal of Engineering Mechanics, 130 (2004) págs. 997-1005.

[24] L. Bruno, C. Canuto, D. Fransos, Stochastic aerodynamics and aeroelasticity of a flat plate via generalised Polynomial Chaos, Journal of Fluids \& Structures, 25 (2009) $1158-1176$.

[25] M.K. Chryssanthopoulos, C. Poggi, Probabilistic imperfection sensitivity analysis of axially compressed composite cylinders, Engineering Structures, 17 (1995) 398-406.

[26] G. Stefanou, The stochastic finite element method: Past, present and future, Computer Methods in Applied Mechanics \& Engineering, 198 (2009) 1031-1051.

[27] Z. Kala, Fuzzy sets theory in comparison with stochastic methods to analyse nonlinear behaviour of a steel member under compression, Nonlinear Analysis Modelling \& Control, 10 (2005) 65-75.

[28] F. Massa, T. Tison, B. Lallemand, A fuzzy procedure for the static design of imprecise structures, Computer Methods in Applied Mechanics \& Engineering, 195 (2006) 925-941.

[29] L.A. Zadeh, Fuzzy sets, Information \& Control, 8 (1965) 338-353.

[30] Z. Qiu, I. Elishakoff, Antioptimization of structures with large uncertain-but-non-random parameters via interval analysis, Computer Methods in Applied Mechanics \& Engineering, 152 (1998) 361-372.

[31] D. Moens, D. Vandepitte, Interval sensitivity theory and its application to frequency response envelope analysis of uncertain structures, Computer Methods in Applied Mechanics \& Engineering, 196 (2007) 2486-2496.

[32] D. Moens, D. Vandepitte, An interval finite element approach for the calculation of envelope frequency response functions, International Journal for Numerical Methods in Engineering, 61 (2004) 2480-2507.

[33] S. Mcwilliam, Anti-optimisation of uncertain structures using interval analysis, Computers \& Structures, 79 (2001) 421-430. 
[34] N. Impollonia, G. Muscolino, Interval analysis of structures with uncertain-but-bounded axial stiffness, Computer Methods in Applied Mechanics \& Engineering, 200 (2011) 1945-1962.

[35] K. Fujita, I. Takewaki, An efficient methodology for robustness evaluation by advanced interval analysis using updated second-order Taylor series expansion, Engineering Structures, 33 (2011) 3299-3310.

[36] I. Elishakoff, P. Elisseeff, S.A.L. Glegg, Nonprobabilistic, convex-theoretic modeling of scatter in material properties, Aiaa Journal, 32 (2012) 843-849.

[37] Y. Benhaim, Convex Models of Uncertainty in Radial Pulse Buckling of Shells, Journal of Applied Mechanics, 60 (1993) 683-688.

[38] Ben-Haim, Yakov, Elishakoff, Isaac, Convex models of uncertainty in applied mechanics, Elsevier1990.

[39] M.S. Chowdhury, C. Song, W. Gao, C. Wang, Reliability analysis of homogeneous and bimaterial cracked structures by the scaled boundary finite element method and a hybrid random-interval model, Structural Safety, 59 (2016) 53-66.

[40] W. Gao, D. Wu, C. Song, F. Tin-Loi, X. Li, Hybrid probabilistic interval analysis of bar structures with uncertainty using a mixed perturbation Monte-Carlo method, Finite Elements in Analysis \& Design, 47 (2011) 643-652.

[41] W. Gao, C. Song, F. Tin-Loi, Probabilistic interval analysis for structures with uncertainty, Structural Safety, 32 (2010) 191-199.

[42] W. Zhang, J. Liu, C. Cho, X. Han, A hybrid parameter identification method based on Bayesian approach and interval analysis for uncertain structures, Mechanical Systems \& Signal Processing, s 60-61 (2015) 853-865.

[43] B. Xia, D. Yu, X. Han, C. Jiang, Unified response probability distribution analysis of two hybrid uncertain acoustic fields, Computer Methods in Applied Mechanics \& Engineering, 276 (2014) 20-34.

[44] C. Wang, Z. Qiu, Y. He, Fuzzy interval perturbation method for uncertain heat conduction problem with interval and fuzzy parameters, International Journal for Numerical Methods in Engineering, 104 (2015) 330-346.

[45] M. Modares, S. Venkitaraman, Reliable condition assessment of structures using hybrid structural measurements and structural uncertainty analyses, Structural Safety, 52 (2014) 202-208.

[46] J.E. Hurtado, D.A. Alvarez, The encounter of interval and probabilistic approaches to structural reliability at the design point, Computer Methods in Applied Mechanics \& Engineering, s 225-228 (2012) 74-94.

[47] J. Guo, X. Du, Reliability sensitivity analysis with random and interval variables, International Journal for Numerical Methods in Engineering, 78 (2009) 1585-1617.

[48] B. Xia, D. Yu, J. Liu, Hybrid uncertain analysis of acoustic field with interval random parameters, Computer Methods in Applied Mechanics \& Engineering, 256 (2013) 56-69.

[49] I. Elishakoff, P. Colombi, Combination of probabilistic and convex models of uncertainty when scarce knowledge is present on acoustic excitation parameters, Computer Methods in Applied Mechanics \& Engineering, 104 (1993) 187-209.

[50] I. Elishakoff, G.Q. Cai, J.H.S. Jr, Non-linear buckling of a column with initial 
imperfection via stochastic and non-stochastic convex models, International Journal of Non-Linear Mechanics, 29 (1994) 71-82.

[51] L.P. Zhu, I. Elishakoff, Hybrid probabilistic and convex modeling of excitation and response of periodic structures, Mathematical Problems in Engineering, 2 (1996) 143-163.

[52] M.J. Zhou, A Design Optimization Method Using Evidence Theory, Journal of Mechanical Design, 128 (2005) 1153-1161.

[53] C. Jiang, W.X. Li, X. Han, L.X. Liu, P.H. Le, Structural reliability analysis based on random distributions with interval parameters, Computers \& Structures, 89 (2011) 2292-2302.

[54] C. Jiang, X. Han, W. Liu, J. Liu, Z. Zhang, A Hybrid Reliability Approach Based on Probability and Interval for Uncertain Structures, Journal of Mechanical Design, 134 (2012) 310-311.

[55] M. Papadrakakis, N.D. Lagaros, Reliability-based structural optimization using neural networks and Monte Carlo simulation, Computer Methods in Applied Mechanics \& Engineering, 191 (2002) 3491-3507.

[56] F. Moses, Problems and prospects of reliability-based optimization, Engineering Structures, 19 (1997) 293-301.

[57] K.H. Lee, G.J. Park, Robust optimization considering tolerances of design variables, Computers \& Structures, 79 (2001) 77-86.

[58] I. Doltsinis, Z. Kang, Robust design of structures using optimization methods, Computer Methods in Applied Mechanics \& Engineering, 193 (2004) 2221-2237.

[59] A. Ben-Tal, A. Nemirovski, Robust Truss Topology Design via Semidefinite Programming, Siam Journal on Optimization, 7 (2004) 991-1016.

[60] J. Lógó, M. Ghaemi, M.M. Rad, Optimal Topologies in Case of Probabilistic Loading: The Influence of Load Correlation, Mechanics Based Design of Structures and Machines, 37 (2009) 327-348.

[61] J. Lógó, New Type of Optimality Criteria Method in Case of Probabilistic Loading Conditions, Mechanics Based Design of Structures and Machines, 35 (2007) 147-162. [62] P. Knoll, S. Reich, Level Set Based Robust Shape and Topology Optimization Under Random Field Uncertainties, Structural \& Multidisciplinary Optimization, 41 (2010) 507-524.

[63] S. Chen, W. Chen, A new level-set based approach to shape and topology optimization under geometric uncertainty, Structural \& Multidisciplinary Optimization, 44 (2006) 1-18.

[64] A. Asadpoure, M. Tootkaboni, J.K. Guest, Robust topology optimization of structures with uncertainties in stiffness-Application to truss structures, Computers \& Structures, 89 (2011) 1131-1141.

[65] P.D. Dunning, H.A. Kim, Robust Topology Optimization: Minimization of Expected and Variance of Compliance, Aiaa Journal, 51 (2013) 2656-2664.

[66] M. Schevenels, B.S. Lazarov, O. Sigmund, Robust topology optimization accounting for spatially varying manufacturing errors, Computer Methods in Applied Mechanics \& Engineering, 200 (2011) 3613-3627.

[67] X. Zhao, W. Song, H.C. Gea, L. Xu, Topology optimization with 
unknown-but-bounded load uncertainty, ASME 2014 International Design Engineering Technical Conferences and Computers and Information in Engineering Conference, American Society of Mechanical Engineers, 2014, pp. V02BT03A053-V002BT003A053.

[68] N. Chen, D. Yu, B. Xia, Z. Ma, Topology optimization of structures with interval random parameters, Computer Methods in Applied Mechanics \& Engineering, 307 (2016) 300-315.

[69] N. Olhoff, J. Du, Introductory notes on topological design optimization of vibrating continuum structures, Topology Optimization in Structural and Continuum Mechanics, Springer2014, pp. 259-273.

[70] B. Xia, D. Yu, An interval random perturbation method for structural-acoustic system with hybrid uncertain parameters, International Journal for Numerical Methods in Engineering, 97 (2014) 181-206.

[71] N. Chen, D. Yu, B. Xia, Unified analysis approach for the energy flow in coupled vibrating systems with two types of hybrid uncertain parameters, Mechanical Systems \& Signal Processing, s 70-71 (2016) 542-556.

[72] M.P. Bendsøe, O. Sigmund, Topology Optimization: Theory, Method and Applications, Handbook of Global Optimization, 34 (2004) 179-203.

[73] N.L. Pedersen, Maximization of eigenvalues using topology optimization, Structural \& Multidisciplinary Optimization, 20 (2000) 2-11.

[74] X. Huang, Y.M. Xie, Evolutionary Topology Optimization of Continuum Structures: Methods and Applications, Wiley2010.

[75] Z. Kang, X. Zhang, S. Jiang, G. Cheng, On topology optimization of damping layer in shell structures under harmonic excitations, Structural \& Multidisciplinary Optimization, 46 (2012) 51-67.

[76] S. Min, N. Kikuchi, Y.C. Park, S. Kim, S. Chang, Optimal topology design of structures under dynamic loads, Structural Optimization, 17 (1999) 208-218.

[77] M.P. Bendsøe, O. Sigmund, Material interpolation schemes in topology optimization, Archive of Applied Mechanics, 69 (1999) 635-654.

[78] N. Olhoff, J. Du, On Topological Design Optimization of Structures Against Vibration and Noise Emission, 2009, pp. 217-276.

[79] N. Olhoff, J. Du, Topological design for minimum sound emission from structures under forced vibration, Topology Optimization in Structural and Continuum Mechanics, Springer2014, pp. 341-357.

[80] V.B. Hammer, N. Olhoff, Topology Optimization with Design Dependent Loads, Topology Optimization with Design Dependent Loads, 2000.

[81] B.-C. Chen, N. Kikuchi, Topology optimization with design-dependent loads, Finite Elements in Analysis and Design, 37 (2001) 57-70.

[82] D.A. Tortorelli, P. Michaleris, Design sensitivity analysis: Overview and review, Inverse Problems in Science and Engineering, 1 (1994) 71-105.

[83] X. Huang, Y.M. Xie, Convergent and mesh-independent solutions for the bi-directional evolutionary structural optimization method, Finite Elements in Analysis \& Design, 43 (2007) 1039-1049.

[84] X. Huang, Y.M. Xie, Bi-directional evolutionary topology optimization of 
continuum structures with one or multiple materials, Computational Mechanics, 43 (2009) 393-401.

[85] C. Jiang, B.Y. Ni, X. Han, Y.R. Tao, Non-probabilistic convex model process: A new method of time-variant uncertainty analysis and its application to structural dynamic reliability problems, Computer Methods in Applied Mechanics and Engineering, 268 (2014) 656-676. 